\title{
Health Equity, Population Health, and Climate Change Adaptation in Ontario, Canada
}

\author{
CHRIS G. BUSE, PhD, University of Northern British Columbia
}

\begin{abstract}
Climate change holds the potential to exacerbate existing health inequalities, yet understanding how practitioners conceive health equity and health equality has received little attention in the scholarly literature. This contribution utilizes in-depth interviews with public health practitioners from health units across Ontario, Canada to characterize understandings of equity in relation to on-going climate change adaptation work. Perceptions of health equity and associated public health practices are described before discussing the resulting implications for how and why practitioners take up the equity agenda in relation to climate change. In doing so, this work problematizes existing public health tools and competencies and signals the emergence of new practices capable of simultaneously promoting adaptive capacity to climate change and reducing health inequity in Ontario.
\end{abstract}

Keywords: Climate change adaptation; health equity; Ontario Public Health Standards; environmental health protection 


\section{Introduction}

Climate change is associated with increasing incidence of heat-related morbidity and mortality, increasingly poor air quality and resulting respiratory conditions, increasing accidental morbidity and mortality attributable to extreme weather - as well as the increasing incidence of mental health impacts post disasters, the spread of vector-borne diseases to previously inhospitable climates, and risks associated with food and water contamination (Health Canada 2005). However, these impacts will not be distributed evenly across the Canadian population, and research suggests that population groups who already experience relatively poor health outcomes compared to national or provincial averages are likely to be differentially impacted, and thus, climate change holds the potential to exacerbate existing health inequalities (Health Canada 2008).

To date, the scholarly literature has primarily focused on climate justice and the health equity implications of climate change for developing countries (Campbell-Lendrum and Corvalan 2007; Ikeme 2003; Kjellstrom et al. 2007), but less attention has been given towards health equity related to climate change in the North American context (Buse 2013). This paper critically examines understandings of health (in)equity and health (in)equality by frontline practitioners. Using empirical data from a case study of climate change adaptation in Ontario, Canada, I analyze and discuss how public health practitioners working on climate change locate health equity in their work, and identify practices related to the promotion of health equity. The discussion problematizes existing tools and approaches for addressing health equity in relation to public health adaptation to climate change, and builds a theoretical case for how health equity discourse can further the field of climate change adaptation in public health.

\section{Situating health equity in the context of public health adaptation to climate change}


The primary focus of North American climate change and health research has thus far sought to document existing vulnerabilities and the uneven health outcomes resulting from physiological sensitivity or differential exposure to climate variability and extreme climate events based on key demographic, social and cultural variables (Berry, Paterson and Buse 2014; Ebi, Kovats and Menne 2006; WHO/PAHO 2012). This literature employs "vulnerability assessments" to identify vulnerable populations according to socioeconomic status, social isolation, and geographic proximity to climate-related hazards. Such assessments indicate that populations more vulnerable to climate change tend to have higher rates of pre-existing chronic conditions or disability and include groups such as the elderly, children, and those experiencing relative socio-economic deprivation or social isolation (Berry and Cheng 2013; WHO/PAHO 2012).

Climate change therefore requires a nuanced understanding of health inequity and health inequality in relation to specific interventions. For example, sharing health risk information about the impacts of climate change — a requirement in the Ontario Public Health Standardswill not serve vulnerable populations equally (Ebi and Semenza 2008). This is primarily because such health promotion activities often fail to incorporate an understanding of how health behaviours and risks are influenced by environmental, socioeconomic and cultural settings (Baum and Fisher 2014).

Promoting health equity has been identified as a central goal of public health practice (Krieger and Birn 1998; Marmot et al. 2008). It has been enshrined in the Canadian public health profession since the development of the Ottawa Charter of Health Promotion and more recently as an underlying value for practitioners in the Public Health Agency of Canada's (2008) Core Competency Statement. A large body of scholarship focuses attention on definitional differences 
between inequalities in health and wellness and inequities (Braveman 2006). Health inequalities largely refer to an unequal distribution or difference in health outcomes between or within populations, whereas health inequity is more specifically concerned with what is right, fair, or just based on whether the cause is unavoidable or unnecessary (Braveman and Gruskin 2003; Kawachi et al. 2002).

Following de Maio (2014), what is fair or moral is ultimately a subjective assessment rooted in deep political values and normative philosophical understandings of morality. While some might argue that this is a semantic difference, the intentional use of the evaluative words “equity" or "inequity", rather than the merely descriptive 'inequality,' invites us to consider the broader social, political, cultural, and environmental contexts that produce health and ill-health in the first place:

If our analysis remains focused on inequality, it becomes distracted by the symptoms of the problem, rather than its determinants. Inequity reflects the essence of the problem; inequality is an empirical measure of inequity made apparent through statistical analysis... Inequality is the observable and collective expression of inequity. Inequalities are measured; inequities are judged (Guzman 2009, p.116).

Nevertheless, it remains unclear how these values are understood, interpreted and embodied by public health practitioners, and how values are translated into concrete action to address the social and environmental determinants of health, particularly in relation to climate change adaptation programming. This paper utilizes a comparative analysis of interviews with public health practitioners working on climate change adaptation initiatives across Ontario, Canada to inform an understanding of the degree to which equity considerations factor into climate change adaptation at the level of regional public health authorities. This paper addresses how health equity is conceptualized by front line public health practitioners in relation to climate change and documents how the meanings associated with equity are translated into practical 
action. The paper ends with a discussion of the theoretical implications these results hold for understanding the discursive construction of emerging fields of public health practice and the role of healthy equity therein.

\section{Research Methods}

This paper is part of a broader study that examined the emergence of climate change adaptation among Ontario health units. The study used a critical realist epistemology informed by Pierre Bourdieu's (1990) 'theory of practice' to understand how some practices in public health are forwarded as more legitimate than others, and to examine the moral and professional commitments of the people responsible for the climate change file across Ontario's 36 regional health units. The primary focus of the investigation was to understand how individuals are able to promote social change within public health organizations in the face of new and emerging fields of public health practice (i.e. climate change adaptation).

I used qualitative semi-structured interviews with employees of Ontario public health units. All 36 health units were invited to participate in a one-hour interview, and research participants were recruited by email from a web-scan where participants were contacted based on whether they were listed as having engaged in climate-related programs or were situated in an environmental health hazards division. In cases where multiple people from a single health unit were identified, I invited that group to select the person they felt was best able to speak to the interview questions. This sampling protocol was designed given that climate change is mandated to be addressed under the environmental health hazards protocol of the Ontario Public Health Standards (2008/2014).

I conducted all data collection, transcription and analysis with formal ethical approval from the University of Toronto Research Ethics Board. Informed consent was obtained in writing 
prior to the interviews, which were conducted by person wherever possible, but primarily over the telephone. Interviews were recorded to ensure the accuracy of collected data. Ultimately, I completed 20 interviews with practitioners from 20 different health units, with 13 health units refusing to participate on the grounds that they did not feel they had engaged with climate change as a public health issue, and three not responding.

Research participants were asked about their personal and professional backgrounds, what their health unit is doing related to climate change adaptation, and how practitioners understand and act on health equity concerns related to climate change. Interviews were transcribed verbatim by the author. Given that climate change is a politically charged topic, pseudonyms were created to ensure anonymity of research participants, and the health units that participated in this research study are also not identified by name.

Additionally, three interpretive analytic tools were iteratively used to explore the data, establish rigor, and develop themes emerging from the data. First, to preserve the contextual uniqueness of each interview and to highlight key findings at the level of individual interview respondents, case summaries were produced. Case summaries are essentially condensed transcripts that identify key individual actions, personal reasons for engaging with equity issues, and other thematic issues that arose during the interview. Given that interpretive decisions were made in terms of what was included or excluded in the case summary, they were not intended to be complete synopses of interview transcripts and were therefore not sent to research participants for validation. Rather, case summaries were simultaneously utilized to reflexively assess the interview process through a language-centred analysis of my own social location in relation to those being interviewed (Mauthner and Doucet 2013). Second, a cross-case display matrix (Miles and Huberman 1994) visually displayed key variables loosely organized around the 
interview questions that largely spoke to organizational responses to climate change. Throughout these processes, extensive qualitative memos were produced in relation to how practitioners conceived the notion of health equity in their climate change work, and what practices specific health units leveled at the equity question. Finally, the analysis utilized memos and the data produced from the first components of the analysis to conduct a form of triangulation called "category zooming" (Halkier 2011) that allows researchers to pursue greater interpretive depth on single points and findings from the broader study. This approach seeks to develop analytic insights, explore counter evidence, and determine how the concept of health equity was taken up by practitioners in the interview context.

\section{Results}

The 20 completed interviews offer a geographically representative sample of Ontario health units when aggregated by census division. A key finding that emerged from interviews was that eight interview participants were innovative champions of climate change work, actively pursuing new practices and new ways of engaging with this issue. The remaining twelve assumed an environmental approach that was oriented towards traditional elements of public health practice including disease monitoring and surveillance.

\section{How do practitioners conceive of health equity in relation to climate change? When asked}

what role health equity plays in climate change adaptation programming and how it informs dayto-day practice, practitioners often asked for clarification, such as "what do you mean by health equity?" or "so what exactly do you mean by that though, like in terms of health equity and the social determinants of health for public health programming?". This may be the result of 
widespread recognition within the public health community of the definitional difference described above. Indeed, half of the research participants accepted, in principle, the importance of the health equity agenda and how it was (or could be) linked to climate change adaptation. In reflecting upon this and wanting to more deeply engage with supposed underlying core values of Canada's public health community, I prompted practitioners to describe their understanding of equity in relation to climate change and to identify any associated programs where both were being addressed.

Findings suggest that health equity is most typically associated with a focus on “vulnerable' populations." Vulnerable populations were often perceived as individuals who are differentially exposed or physiologically sensitive to climate-related health impacts (e.g. seniors, children and attendees of outdoor events are all more susceptible to the physiological impacts of extreme heat; new immigrants may face language barriers in terms of understanding and adapting to extreme weather events). However, health equity was most typically associated with poverty:

I mean we've got, and I shouldn't say this...but we're poor! [laughs] We have vulnerable populations. I mean it's not an issue that is disappearing. We have real hard case poverty rates [in this area] that are above the Ontario average and we are aware of this.

Both champion practitioners and non-champion practitioners often conceived of health equity as measurable differences in resource access or distribution between or within population groups and how those differences manifest in terms of disparate health outcomes through monitoring and surveillance.

Several practitioners went on to add that populations living in poverty may be doubly disadvantaged because low incomes are often associated with poor housing or living in areas that may be differentially exposed to extreme weather events (e.g. living in a flood plain). For 
example, one research participant claimed "there is a bit of a tendency for poorer people to live in lower lying areas. I mean that's not a universal thing, but there is definitely a tendency there."

This was echoed by other research participants as well:

We know that, for example, for flooding, the areas of [laughing], like most areas in most flooding events, they tend to impact the areas of town where...the population with the lowest income tend to live. With the poorest housing stock. That type of thing. And, so, we do know that we have like this, um, people with low socioeconomic status and the most marginally housed people are the people that are most likely to be affected by flooding.

The common focus on poverty and multiple layers of social or economic disadvantage was not surprising given that practitioners also often evoked the discourse of the social determinants of health to contextualize their thinking around health equity:

As far as health equity advocacy work and different strategies and activities, and in terms of bigger projects and plans to address all that. You know, that's a priority identified again within health hazards.

In generalities, the health department is very well aware of health equity issues and social determinants of health and in my mind the two are linked, though I know they are a little bit different. Um, we do have a couple of people working on both, I'm going to say both, I might not be right about this, but certainly the social determinants of health and as I mentioned what flows out of those kinds of things or issues around health equity.

The quotes above not only exemplify how provincial policy and the values of specific health units inform understandings of equity, but they also signal that health equity exists as its own subfield of public health practice with its own meanings and cultural competencies, some of which may overlap with climate change activities.

For example, some practitioners took a more nuanced view of equity issues in relation to climate change. Specifically, the champions of climate change adaptation work tended to utilize the language of "differential exposure", "sensitivity" and "adaptive capacity" to describe their activities. This language is explicitly related to climate impacts and adaptation which may be 
unfamiliar to those who do not have a working knowledge of the emerging field, but which

seems to be used by practitioners to bridge the sub-fields of climate change adaptation and health equity:

Our poor are the ones that are least able to cope with it so we analyze it from an exposure perspective, as I said. Poor people, vulnerable people, people that aren't able to protect themselves, could be exposed differently which is an issue of location, urban vs. rural in our area and their individual susceptibilities or sensitivity, call it what you want.

This proposed model that I've developed to reduce vulnerability is something that embeds some of the familiar terms and some of the familiar approaches and processes that public health typically is accustomed to, with the exception of the whole vulnerability piece: the sensitivity, exposure, adaptive capacity. Those are new terms that I need some work to do to educate on. But, you know, just presenting this, I've actually, you know, gone around informally and presented this to various departments...And it really resonates.

The use of climate-specific language for describing health equity is aligned with existing scholarly work on climate change and health. Its discursive use helps to further contextualize the relatively new and emerging nature of climate change adaptation in the public health sector.

However, it should be noted that this language for describing differential health outcomes is still heavily steeped in a definition of equity that privileges distributional health inequalities or disparities over considerations of fairness or justice in the conditions that lead to those disparities in the first place. Moreover, the methodology employed to identify health vulnerabilities is very much rooted in epidemiological principles of monitoring and surveillance (WHO/PAHO 2012), with the hopes that such activities will lead to interventions. This approach is therefore reactionary rather than precautionary. To build on these arguments further, I contextualize these understandings of health equity by identifying the practices associated with climate change adaptation that have an explicit equity focus, as identified by interview participants. 


\section{What are the specific practices that comprise the field of climate change adaptation}

in relation to health equity? The fact that there was little variation between research

participants in terms of their description of equity issues in relation to climate change is unique

insofar as there are key differences in how program development and service delivery link health equity and climate change adaptation across Ontario health units.

An emerging focus on heat extreme heat preparedness and risk communication. The most common association identified between climate change and health equity manifested in terms of heat alert and response systems (HARS) — response plans that trigger a range of activities aimed at mitigating heat exposure in the general population when a certain temperature is reached. It is worth noting that practitioners likely evoked heat-related programs primarily because it is an area that is most tangibly or logically understood in relation to climate change. Heat programming is also mandated in the Ontario Public Health Standards (2008/2014).

However, there is nuance in how HARS are delivered across health units. In more populated areas, health units run a suite of programs including the implementation of cooling centres in public spaces, piloting cooling spaces in apartment buildings, and the dissemination of risk information to the public. In smaller, rural health units which have less staff and resources dedicated to day-to-day logistics, HARS typically consist of basic outreach activities to communicate the health risks associated with exposure to extreme heats, as well as disseminating strategies to avoid overheating:

So I think by that way, we're achieving a level of health equity by spreading the message in a way that is, you know, it's not just us that's saying it, we're sending it to, it's like train the trainer kind of thing where you send it to different groups and then they deal with it in, with people they work with. 
The practice of communicating risk in an attempt to bolster adaptive capacity can, however, be problematized in the context of a relatively robust literature that challenges the degree to which behaviour change is possible, particularly for groups that experience systemic disadvantages (Baum and Fisher 2014). The communication of health risks further gives the illusion that health units are doing something on climate adaptation issues, while simultaneously downloading the responsibility for ill health outcomes to other agencies or to the public themselves. There are also additional challenges for evaluating who uses this kind of health information and to what end, and whether priority populations are reached at all. For example, several champion practitioners expressed concern that communicating heat information may be culturally incompatible with some populations in their community. When probed on a cooling centre pilot project for apartment buildings, one champion noted that:

There were some cultural aspects of a room that some groups didn't like to mix or felt uncomfortable. Other people felt that they would go other places. So they would go visit their friends. Unfortunately, some of the real vulnerable people just stayed in their apartments because they didn't have the ability to get down to a cooling room.

Champions were also quick to point out the limitations of existing risk communication approaches and the documenting and monitoring of so-called vulnerable populations. These practitioners had a keen awareness of the stigmatizing impacts of labeling a particular community as vulnerable and were actively struggling with how to intervene in a way that was equitable and fair:

So for example, if we identify that low socio-economic status is a risk factor for exposure to extreme heat and then we start mapping those locations of low socioeconomic status, some municipalities may not want that information to be identified on a map. Whether they feel it's going to stigmatize that neighbourhood or it might affect their economic development if they're trying to bring growth into their communities. So we're kind of struggling with on one hand, we need to protect the public and we need to target where that, the public is most vulnerable. How do we reach that target population if we're not 
allowed to show that information and share that with our municipalities? So right now we are having an internal conversation to address that challenge.

Other promising practices for integrating health equity in climate change adaptation. By virtue of occupying larger health units with more staff and resources, champions may be better equipped to attune themselves to equity issues in their communities. Moreover, by virtue of having resources to focus on new and potentially innovative program design, well-resourced health units may be able to more actively engage with climate change adaptation programming more generally. For example, one Medical Officer of Health suggests that the burgeoning focus of Ontario health units on built environment issues (i.e. promoting walkable and 'green' communities) are a meaningful way of addressing equity:

Certainly the built environment piece overlaps explicitly with health equity. In our literature review, for example, we looked at the potential for built design to address poverty issues and found that mixed neighbourhood issues are important for that...The complete, complete communities and complete streets approach helps everybody, including and probably in particular, including people of lower income.

This focus on the built environment also required increased collaboration with regional and city planners to ensure that communities are being developed in a sustainable and health promoting manner was corroborated with other practitioners. This was further evidenced in interviews by positioning the focus on the built environment to mitigate urban heat islands.

Moreover, at least four Ontario health units - the majority of which are located in larger metropolitan areas and have larger staff, budgets, and more resources - developed climate change and health vulnerability assessments specific to their regions which provide baseline epidemiologic surveillance and monitoring of specific populations who may be more vulnerable to specific health impacts. Three others developed various forms of health impact assessments related to heat, vector-borne diseases, and extreme weather events. These approaches primarily 
resorted to using disease monitoring and surveillance data to describe current vulnerability and project future vulnerability. As suggested above, such assessments were largely driven by the fact that these organizations tended to employ health equity as a discursive tool to frame a broad array of strategic priorities and utilize the goals of a pre-existing subfield of public health practice to engage in other activities on the fringes of more conventional practice. However, given the novel nature of the vulnerability assessment approach and the fact that these practices are early on in their tenure, few programs have resulted from this form of surveillance and monitoring and further research may be needed to determine the utility of vulnerability assessments in producing practices that are capable of eliminating or reducing health disparities, rather than just documenting them as a baseline.

Rural and urban health units have varying capacity to address climate change adaptation. The findings above indicate that there are a variety of health equity programs linked to climate change adaptation across the province, but that some health units are so under-resourced they lack the capacity to even engage in the communication of environmental health hazards with vulnerable populations, or have yet to complete this work but are planning on incorporating this type of program in the future. For instance, one research participant indicated that cooling centres are an important element to incorporating health equity considerations into climate change adaptation programming:

You know, I think heat alerts, working with municipalities about heat alerts and having as part of that their consideration about cooling centres would be particular[ly] important for people of lower income who don't have access to air conditioning, right? So we, this is speaking to intentions, we haven't gone on with this, but we intend to go on with this type of thing. So there you're getting into health equity and climate change adaptation explicitly. 
A lack of programming targeting heat was mirrored with a general low level of focus on climate change in predominantly rural or northern health units. Several practitioners identified health equity as a strategy within their health hazards division, but almost all health units had limited resources to engage with climate change and related equity concerns. This was largely identified as being a product of how health units are funded across Ontario (a mix of provincial and municipal/regional government funding). Thus, health units with a larger constituency have a larger tax base to draw from, and accordingly, have more staff and funding to pursue innovative activities. This is in contrast to rural and remote health units that utilize their limited resources to address what they identify as priority health concerns throughout their respective regions.

Climate change already takes a back seat in terms of staff and resources with the programs that we all have to do, and then within that program we're talking about the environmental soils study report and the housing situation here, in terms of mold complaints, etc. And then with the rest of that, if there's any room leftover we, if we were to contemplate climate change and radon and indoor air quality, outdoor air quality, etc."

At this point, it is important to identify that the champions of climate change adaptation work were almost exclusively employed by health units in the Greater Golden Horseshoe region of Ontario. It is not surprising that the Greater Golden Horseshoe is a central hub of climate change adaptation for public health. This area comprises the majority of Ontario's population and accordingly there are a dense array of social service and environmental organizations working at the nexus of climate change and equity issues. Indeed, several research participants indicated unfavorable comparisons to Toronto Public Health in terms of geography, density and access to resources: "We're not like Toronto at all and we can't be, because we are predominantly rural...We're not the same....and there are a lot of other agencies in Toronto that depend on those heat alerts to then initiate their action plans". 
Equity as a guiding concept for climate change practice. Equity issues were identified as a guiding concept to inform the overall practice of a health unit for both champions and those who assumed a more conventional environmental health approach to engaging climate change. For instance, an associate medical officer of health--before describing their health unit's HARS-first provided context to the equity component of their work by positioning it in relation to a vulnerable population plan which provides emergency outreach to vulnerable populations across all hazards:

Our vulnerable populations plan is all hazards, right? It's not just heat. And it's also, again, a way of reaching vulnerable groups in whatever situation that's required... So, we cross-reference that plan with our heat messaging, and so all our groups dealing with vulnerable populations will receive that kind of heat messaging as well.

Similarly, one champion who was responsible for conducting his health unit's climate change and health vulnerability assessment indicated that equity was a strategic priority, but not in relation to the on-going climate change adaptation work.

However, most champions of climate change adaptation who explicitly discussed the strategic positioning of climate change and health equity work together in an attempt to identify common ground and synchronize research and practice agendas:

So [health equity] really is an underlying area that we have focused on especially in the last five years. [Our Medical Officer of Health] has really seen equity issues as being a priority issue. We still do broad based public health, provide some messages out to the general public, but we really see that the most vulnerable are the ones that are the most impacted by various health determinants.

Thus, not only is health equity its own subfield of practice, but practitioners, and champions in particular utilize the discourse of health equity to lend symbolic capital and 
credibility to their climate change adaptation work. In attempting to address existing vulnerabilities and disparities in health outcomes, climate change champions tended to evoke health equity as a legitimate entry point into climate change adaptation work. Health equity was therefore employed as a discursive strategy by champions to "bridge the gap that exists across the province" on climate change adaptation options. The simultaneous acknowledgement of existing health equity concerns, when coupled with the fact that climate change can exacerbate those conditions, also tended to resonate more highly with senior decision-makers, thereby opening up possibilities for more innovative actions than conventional risk communication.

\section{Discussion: Health equity as discourse vs. health equity as field}

The way in which equity is defined and understood by public health practitioners led to a relative absence of critical considerations of health equity as an explicitly moral goal to be pursued by the public health community. This in turn led practitioners to equate health equity with health disparities. Semantically, inequalities or disparities in health result from inequities. As demonstrated above, defining equity as health disparities with little consideration of whether those differences are moral or just engenders particular responses (i.e. risk communication) with the goal of balancing health outcomes for vulnerable populations - a goal which may be unachievable if the root causes of inequalities such as poverty and the conditions that maintain it remain unaddressed.

However, taking a deeper look at the practices described by practitioners in addressing health equity and climate change adaptation suggests that the champions of this work may be more proactive in addressing equity issues in their communities than are those practitioners with more conventional public health dispositions. However, this is evident through their climate 
change adaptation practice, rather than their definitional understanding of 'equity' or 'equality'. Despite a common focus on population health disparities, champions tended to pursue activities to guide adaptation at the local level in ways that were more innovative and focused on prevention. Moreover, the champions appear to be more proactive in identifying and leveraging financial and staff resources to address health equity issues. Conversely, practitioners who did not use equity as a concept to help guide their work tended to highlight their health unit's existing risk communication programs and a lack of resources to implement additional programming.

The ways in which equity work is practiced was nuanced, but appeared to be most often related to health unit budgets and resource allocation. In some cases, but primarily in the Greater Golden Horseshoe, equity served as a guiding concept to influence climate change adaptation as well as a multitude of pre-existing programs. In other cases, health equity was identified as a guiding concept for a variety of public health activities, only not for climate change. For others still, the equity discourse was absent entirely, or had not been given ample attention due to the day-to-day activity needs of the community. Thus, there are very real equity issues between health units across the province and the implications this has for programming between smaller and larger health units.

Thus, the champions of this work, by virtue of primarily occupying larger health units, tend to be better equipped to attend to health equity issues. Findings also serve to further illustrate how the Ontario Public Health Standards (2008/2014)—which mandates health units to communicate the health risks of climate change among other actions - are not necessarily translated into practice by all health units. Moreover, I suggest that practitioners strategically employ health equity as a discourse from which to legitimize additional actions related to climate 
change, whereas health equity—as a strategic priority identified by health units across Ontariohas also become a field of practice in its own right oriented towards the social determinants of health and eliminating health disparities.

As a discursive field (Snow 2008), health equity becomes a strategy employed by actors to align existing goals and values of public health practice with emerging logics and rules of climate change adaptation. This is likely due to the fact that the field of health equity has acquired vast amounts of symbolic capital in the broader field of public health over the last 30 years. With the explosion of interest and action on the social determinants of health, health equity has a degree of legitimacy which climate change adaptation as an emerging field may lack. Accordingly, the practices associated with health equity (e.g. surveillance and monitoring of vulnerable populations; information campaigns designed to improve health behaviours of the most marginalized) can be reproduced on the field of climate change adaptation because they already have a degree of professional credibility, and practitioners are already adept at implementing such activities. These approaches place the onus of responsibility on the individual rather than addressing systemic factors that produce ill-health conditions in the first place. Thus, the existing discourse promotes particular understandings of equity and associated practices, many of which are familiar to practitioners and build on existing public health competencies of monitoring, surveillance and risk communication. The discourses are prescriptive; how they are framed engenders the resulting practical responses. Insofar as new practices emerge from the strategic employ of the health equity discourse, results here therefore suggest that the discourse is also simultaneously representative of a sub-field of practice which overlaps with the emerging field of climate change adaptation. Practitioners borrow logics and discourses from the field of health equity as a way to legitimate emerging practices in others which also serve to reinforce 
dominant understandings of health equity. The relatively durable dispositions of actors trained in public health and the structuring nature of the rules for understanding health equity in the broader field of public health have reproduced an understanding of health equity in the sub-field of climate change. Instead of utilizing climate change as an opportunity to engage in moral discussions of disparate health outcomes between groups, the emphasis on documenting vulnerabilities and communicating health risks is established as both normative and legitimate.

Interventions targeting the health impacts of climate change for priority populations, such as new surveillance and monitoring activities and HARS represent borrowed logics from other sub-fields of public health practice, including health equity. From a practice theory perspective, these findings lend credence to the notion that practices are imbued with materials, competencies, and meanings, and that those meanings are telling of the motives of actors. Whether conscious or subconscious, the practices discussed here are representative of both the structuring nature of the field of public health, and the agency of actors attempting to influence the health of vulnerable communities.

The power of cultural and professional competence is therefore wielded by practitioners who are able to further legitimate particular ways of 'doing' public health. The implications for social change are that practices are re-conceptualized with new language, but constantly reproducing old meanings and recycled logics. This is not to say that nothing is new or innovative about the climate change and health equity agenda in Ontario. For the first time in Ontario's public health history, practitioners are being forced to face uncomfortable questions about how to combine fields of practice in ways that are commensurate with the values of the profession, the goals of the communities they represent, and the interaction between a hierarchical society rife with inequalities and the reality of our changing climate. Practices will 
therefore continue to evolve as the meanings associated with them change. This is evidenced by early adopters in the Greater Golden Horseshoe region of Ontario conducting vulnerability assessments, promoting green and walkable communities, entering into discussions about sustainability within their organizations and out in their communities, contributing ideas to official plans, and revitalizing emergency response protocols in light of increasingly severe weather patterns. These are all profound and positive changes in our efforts to begin adapting to climate change.

However, this article suggests this change to be a slow and necessarily iterative process, at least for the field of public health. Despite health equity and climate change both being areas of policy priority identified by the provincial government, many health units still lack the organizational capacity or the political will to meaningfully address the root causes of health equity issues in their communities, let alone create a suite of potentially transformative practices associated with the field of climate change adaptation, a field which is still slowly gaining a foothold in many parts of the province. Thus, legacies of public health's history continue to inform how we understand environmental hazards and what to do about them. This is not necessarily a negative process, but reproducing reductionist ways of thinking in an attempt to fit linear cause and effect relationships on complex health issues may be ill-suited to addressing multiple and compounding layers of social disadvantage. The importance of furthering the preventative nature of public health action on climate change is therefore required from both the champions of this work, as well as their 'conventional' environmental health practitioner colleagues.

\section{Conclusion}


Employing an equity analysis on this collection of interviews has served to contextualize how health equity is understood by public health practitioners working on climate change adaptation in Ontario, Canada. This analysis raises concerns about operational ability to engage with vulnerable populations, drawing attention to the broader political economy of resource distribution within and between health units and the resulting differences in practices with an explicit equity component. This work also lends support for insights into the change process of practices within public health, how fields emerge, and how their boundaries are contested or reinforced through practice.

Much of the health equity literature suggests that the public health community must entertain conversations about what is moral, fair, and just for the health of the public. Engaging in this discussion requires practitioners to go beyond simply documenting and describing unequal health outcomes. Rather, an equity lens involves addressing the root causes of inequality, a task which frontline professionals may be under-prepared to engage in given the root causes of climate-related health outcomes are outside of the conventional realm of public health activities. Thus, drawing in other actors, leveraging unique policy resources, and being staunch advocates of a moral agenda rooted in justice and fairness may be required. New tools such as adaptive management, collaboration with new allies (e.g. conservation authorities, planners), and applying greater local political pressure to existing systems that generate many of the health inequities that we see today will be necessary to pursue such an agenda. 


\section{References}

Baum, F., \& Fisher, M. (2014). Why behavioural health promotion endures despite its failure to reduce health inequities. Sociology of Health \& Illness, 36(2), 213-225. http://doi.org/10.1111/1467-9566.12112.

Berry, P., Paterson, J. A., \& Buse, C. (2014). The assessment of vulnerability to the health impacts of climate change in Middlesex-London. City of London: Middlesex-London Health Unit. Retrieved from https://www.healthunit.com/uploads/assessment-ofvulnerability-to-the-health-impacts-of-climate-change-in-middlesex-london.pdf.

Bourdieu, P. (1990). The Logic of Practice (Trans. R. Nice). Cambridge: Polity Press.

Braveman, P. (2006). Health Disparities and Health Equity: Concepts and Measurement. Annual Review of Public Health, 27(1), 167-194.

http://doi.org/10.1146/annurev.publhealth.27.021405.102103.

Braveman, P., \&Gruskin, S. (2003). Defining equity in health.Journal of Epidemiology and Community Health, 57(4), 254-258. http://doi.org/10.1136/jech.57.4.254.

Buse, C. (2013). Intersectoral action for health equity as it relates to climate change in Canada: contributions from critical systems heuristics. Journal of Evaluation in Clinical Practice, 19(6), 1095-1100. http://doi.org/10.1111/jep.12069.

Campbell-Lendrum, D., \&Corvalán, C. (2007). Climate Change and Developing-Country Cities: Implications For Environmental Health and Equity. Journal of Urban Health, 84(S1), 109_ 117. http://doi.org/10.1007/s11524-007-9170-X.

Cheng, J. J., \& Berry, P. (2013). Development of key indicators to quantify the health impacts of climate change on Canadians. International Journal of Public Health, 58(5), 765-775. http://doi.org/10.1007/s00038-013-0499-5. 
Crossley, N. (2003). From Reproduction to Transformation Social Movement Fields and the Radical Habitus.Theory, Culture \& Society, 20(6), 43-68. http://doi.org/10.1177/0263276403206003.

Ebi, K. L., Kovats, R. S., \&Menne, B. (2006).An Approach for Assessing Human Health Vulnerability and Public Health Interventions to Adapt to Climate Change.Environmental Health Perspectives, 114(12), 1930-1934. http://doi.org/10.1289/ehp.8430.

Ebi, K. L., \&Semenza, J. C. (2008).Community-Based Adaptation to the Health Impacts of Climate Change.American Journal of Preventive Medicine, 35(5), 501-507. http://doi.org/10.1016/j.amepre.2008.08.018.

Guzman, R. G. (2009). Latin American Social Medicine and the Report of the WHO Commission on Social Determinants of Health.Social Medicine, 4(2), 113-120.

Halkier, B. (2011). Methodological Practicalities in Analytical Generalization.Qualitative Inquiry, 17(9), 787-797. http://doi.org/10.1177/1077800411423194.

Health Canada. (2005). Climate Change: Preparing for the health impacts. Health Policy Research, 11, 1-51.

Ikeme, J. (2003). Equity, environmental justice and sustainability: incomplete approaches in climate change politics. Global Environmental Change, 13(3), 195-206. http://doi.org/10.1016/S0959-3780(03)00047-5.

Kawachi, I., Subramanian, S. V., \& Almeida-Filho, N. (2002).A glossary for health inequalities.Journal of Epidemiology and Community Health, 56(9), 647-652. http://doi.org/10.1136/jech.56.9.647.

Kim, J. Y., Millen, J. V., Irwin, A., \&Gershman, J. (Eds.). (2002). Dying For Growth: Global Inequality and the Health of the Poor (1st edition). Monroe, Me: Common Courage Press. 
Kjellstrom, T., Friel, S., Dixon, J., Corvalan, C., Rehfuess, E., Campbell-Lendrum, D., ... Bartram, J. (2007). Urban Environmental Health Hazards and Health Equity.Journal of Urban Health, 84(S1), 86-97. http://doi.org/10.1007/s11524-007-9171-9.

Krieger, N., \&Birn, A. E. (1998). A vision of social justice as the foundation of public health: commemorating 150 years of the spirit of 1848. American Journal of Public Health, $88(11), 1603-1606$.

Maio, F. D. (2014). Global Health Inequities: A Sociological Perspective. Palgrave Macmillan. Marmot, M. (2007). Achieving health equity: from root causes to fair outcomes. The Lancet, 370(9593), 1153-1163. http://doi.org/10.1016/S0140-6736(07)61385-3.

Marmot, M., Friel, S., Bell, R., Houweling, T. A. J., Taylor, S., \& Commission on Social Determinants of Health. (2008). Closing the gap in a generation: health equity through action on the social determinants of health. Lancet (London, England), 372(9650), 16611669. http://doi.org/10.1016/S0140-6736(08)61690-6.

Mauthner, N. S., \&Doucet, A. (2003).Reflexive Accounts and Accounts of Reflexivity in Qualitative Data Analysis.Sociology, 37(3), 413-431. http://doi.org/10.1177/00380385030373002.

Miles, M. B., \& Huberman, A. M. (1994).Qualitative Data Analysis: An Expanded Sourcebook. Location: SAGE.

Ministry of Health and Long-term Care. (2008/2014). Ontario Public Health Standards. Toronto, ON: MOHLTC. Available at: http://www.health.gov.on.ca/en/pro/programs/publichealth/oph_standards/ (Accessed, July $2,2015)$ 
Public Health Agency of Canada.(2008). Core competencies for public health in Canada (Release 1.0). Ottawa: Minister of Health. Available at: http://www.phac-aspc.gc.ca/phppsp/ccph-cesp/pdfs/cc-manual-eng090407.pdf (Accessed June 23, 2015).

Seguin, J. (2008). Human Health in a Changing Climate: A Canadian Assessment of Vulnerabilities and Adaptive Capacity. Ottawa: Health Canada.

World Health Organization/Pan American Health Organization (WHO/PAHO). (2012).

Protecting health from climate change: Vulnerability and adaptation assessment. Geneva: WHO. 